\title{
Effect of a Pre-Treatment Educational Video in Improving Patient Satisfaction with 5-Fluorouracil Treatment for Actinic Keratoses: A Randomized Controlled Trial
}

\author{
Oma N. Agbai (D) Parastoo Davari · Jennifer Johnson · April Armstrong •
}

Nasim Fazel

Received: April 25, 2016/ Published online: October 24, 2016

(c) The Author(s) 2016. This article is published with open access at Springerlink.com

\begin{abstract}
Introduction: Patient treatment satisfaction and adherence may be affected by the initial understanding of outcomes in the treatment of actinic keratoses with 5-fluorouracil 5\% cream (5-FU). Pre-treatment educational videos may optimize this understanding. The objective of this study was to determine whether prospective patient viewing of an educational video delineating treatment effects and expectations improves patient satisfaction and treatment completion rates for the treatment of actinic keratoses with 5-FU.
\end{abstract}

Enhanced content To view enhanced content for this article go to http://www.medengine.com/Redeem/2E07 F0600C814195.

O. N. Agbai $(\varangle) \cdot$ P. Davari · N. Fazel

Davis Department of Dermatology, University of

California, Sacramento, CA, USA

e-mail: onagbai@gmail.com

J. Johnson

Independent Consultant, Statistical Analysis,

Sedalia, CO, USA

A. Armstrong

Department of Dermatology, University of Southern

California, Los Angeles, CA, USA
Methods: Forty-four participants were recruited to the UC Davis Dermatology outpatient clinic. Each participant was randomized to the video (group A) or control group (group B), and topical 5-FU cream treatment was conducted for 2 weeks in both groups.

Results: A follow-up questionnaire was performed to assess patient satisfaction and adherence to the treatment regimen. The results of these questions were analyzed using the Mann-Whitney test. One item on the questionnaire asked the patient to rate their overall level of satisfaction on a score of 0-100. The results of this question were analyzed using the unpaired $t$ test. The results of the statistical analysis show no significant difference between the patient group that viewed the video and the patient group that did not view the video.

Conclusions: We speculate that this study may establish a foundation for subsequent studies that may affect the broader medical community and promote development of educational videos.

Keywords: 5-fluorouracil; Actinic keratosis; Dermatology education; Patient education; Treatment video 


\section{INTRODUCTION}

Patient understanding and recall of medical instructions have a significant impact on the success of medical treatment. Makki and colleagues demonstrated that written postoperative instructions resulted in decreased discomfort, consumption of fewer analgesics and a more prompt resumption of regular activities [1]. In addition, studies have demonstrated that educational videos are beneficial in the improvement of melanoma awareness [2], promotion of sunscreen use [3] and facilitation of chronic disease management [4]. Educational videos have also shown utility in informed consent proceedings for surgical procedures [5-7].

Topical 5-fluorouracil (5-FU) is an effective treatment for actinic keratoses [8]. Despite its widespread use, patient satisfaction rates are less than optimal. In a questionnaire study comparing multiple topical therapies for actinic keratoses, 38\% of patients were "extremely or very satisfied" with topical 5-FU therapy, while $61 \%$ were "somewhat," "minimally" or "not satisfied" with treatment [9]. Poorly defined treatment expectations and long treatment courses may contribute to low patient satisfaction following treatment with 5-FU. Viewing a pre-treatment educational video may provide optimized preparation for effects of treatment, which may improve patient satisfaction and treatment completion rates with 5\% 5-FU for treatment of actinic keratoses. The present study aims: (1) to determine whether a pre-treatment video delineating treatment effects and expectations improves patient satisfaction and treatment completion rates, compared to usual verbal instruction, regarding administration of topical 5\% 5-fluorouracil cream for the treatment of actinic keratosis involving the face, scalp, upper chest, dorsal hands and forearms.

\section{METHODS}

A total of 44 participants were recruited to the UC Davis Dermatology outpatient clinic. The institutional review board at this site approved the research protocol. Each participant presented for a regularly scheduled office visit for AKs needing field therapy such as 5-FU. Each participant signed an informed consent form and was randomized to the video or control group though a standardized randomization process. A participant randomized to the video group (group A) viewed a brief video on 5-FU treatment (physician absent) after a brief introduction to 5-FU treatment by the MD provider. The participant in the control group (group B) received the usual verbal instruction on 5 -FU treatment and had all questions answered by the MD provider as would be done in a standard dermatology office visit. For both interventions, the proper use and potential adverse effects of the treatment were discussed. The video also included clinical images of expected treatment effects during treatment. The treatment for 5-FU cream began within 3 weeks of enrollment. The participant was instructed to apply topical 5-FU cream twice daily for 2 weeks. The follow-up questionnaire (Table 1) was performed via telephone and completed within 6 weeks of completing treatment. The ten-item questionnaire was designed to assess participant satisfaction and treatment adherence.

In this pilot study, the number of subjects (22 per study arm, 44 total) was based on data from a study by Tierney and colleagues [9] in which 45 patients were surveyed in a study evaluating patient satisfaction with various 
Table 1 Post treatment questionnaire for groups A and B

1. What is your overall level of discomfort during treatment with cream:
a. None
b. Minimal
c. Moderate
d. Severe

2. How long did it take for your skin to return to "normal" after completing the 5-FU treatment:
a. Less than 7 days
b. 7 to 14 days
c. 14 to 21 days
d. More than 21 days

3. How much did the 5-FU treatment disrupt your everyday life?
a. Significantly
b. Somewhat
c. Slightly
d. Not at all

4. How do you feel about the appearance of your skin after 5-FU treatment?
a. Much improved
b. Improved
c. Unchanged
d. Worse

5. How do you feel about the overall effectiveness of the 5-FU treatment?
a. Very effective
b. Moderately effective
c. Slightly effective
d. Not effective

6. How satisfied are you with the doctors who treated your pre-cancers (actinic keratoses)?
a. Very satisfied
b. Somewhat satisfied

Table 1 continued
c. Minimally satisfied
d. Not satisfied

7. How likely are you to recommend this treatment to others with similar skin conditions?

a. Would strongly recommend

b. Would recommend with some confidence

c. Would recommend with hesitation

d. Would not discuss

e. Would discourage others from using this treatment

8. Did you complete your treatment as instructed by your dermatologist? Choose one of the following:

a. I applied the cream every single day of treatment

b. There are 1 to 3 days when I missed at least one dose

c. There are 4-7 days when I missed at least one dose

d. There are over 7 days when I missed at least one dose

e. I stopped the treatment early (please state reason why treatment was stopped below):

9. Please rate your level of understanding of what to expect during and after treatment with Efudex:

a. Excellent

b. Very Good

c. $\mathrm{Ok}$

d. Poor

10. What is your overall level of satisfaction with the 5-FU treatment? Please provide a score from 0 to 100 , with 0 being the worst and 100 being the best

treatment options for actinic keratoses. Of the original 45 subjects enrolled, 39 returned the mail-in questionnaires with an 86.7\% completion rate. The researchers found that photodynamic therapy had equivalent or improved recovery times, cosmetic outcomes, patient satisfaction and preference as a treatment for actinic keratoses when compared 
to other treatment options. Although ours is a pilot study, we would like to note that these results reached statistical significance.

Our study had $90 \%$ power to detect a $1-\mathrm{cm}$ difference in satisfaction on a $10-\mathrm{cm}$ visual analog scale with $\alpha=0.05$ if 20 subjects were recruited in each group. We then accounted for $10 \%$ subject dropout and therefore recruited 22 subjects for each treatment arm for a total of 44 total subjects. The chosen effect size represented clinically meaningful difference based on both the prior literature and its comparability to seven-point categorical satisfaction scales.

The results of a patient satisfaction survey were analyzed to evaluate the hypotheses $\mathrm{H}_{\mathrm{O}}=$ patient satisfaction of treatment does not increase with the educational video vs. $\mathrm{H}_{1}=$ patient satisfaction of treatment does increase with the educational video. A survey of 10 questions was administered to 44 participants; 22 had watched the video, and 22 had usual verbal instruction. Thirty participants completed the survey, though not all were completed in entirety; $68 \%$ of the patients responded to the survey, $64 \%$ of group A and $73 \%$ of group B. This response rate was slightly less than the allotted $10 \%$ drop-out rate per the research plan. Responses for individual questions ranged from $n=28$ to $n=30$. Questions 1 through 9 were categorical responses measuring patient satisfaction of treatment. The results of these questions were analyzed using the Mann-Whitney test. Question 10 asked the patient to rate their overall level of satisfaction on a score of 0 to 100 . The results of this question were analyzed using the unpaired $t$ test. If the sample size had been larger it would have been beneficial to compute a chi-square test to determine odds ratios for categorical responses.

\section{Compliance with Ethics Guidelines}

All procedures followed were in accordance with the ethical standards of the responsible committee on human experimentation (institutional and national) and with the Helsinki Declaration of 1964, as revised in

Table 2 Statistical analysis

\begin{tabular}{|c|c|c|c|c|c|c|c|c|c|}
\hline \multicolumn{10}{|c|}{ Mann-Whitney B test } \\
\hline Question & $P$ value & $\mathbf{n A}$ & $\mathrm{nB}$ & MedianA & MedianB & $25 \% \mathrm{~A}$ & $25 \% \mathrm{~B}$ & $75 \% \mathrm{~A}$ & $75 \% \mathrm{~B}$ \\
\hline Ql & 0.432 & 14 & 15 & 2.5 & 3 & 1 & 2 & 3 & 3 \\
\hline Q2 & 0.207 & 12 & 16 & 2 & 2 & 2 & 1 & 3 & 2 \\
\hline Q3 & 0.176 & 14 & 16 & 4 & 3 & 2 & 2 & 4 & 4 \\
\hline Q4 & 0.446 & 14 & 16 & 2 & 2 & 1 & 1 & 3 & 2 \\
\hline Q5 & 0.669 & 13 & 16 & 2 & 1.5 & 1 & 1 & 2.5 & 2 \\
\hline Q6 & 0.209 & 14 & 16 & 1 & 1 & 1 & 1 & 1 & 1 \\
\hline Q7 & 0.907 & 13 & 16 & 2 & 2 & 1 & 1 & 2 & 2 \\
\hline Q8 & 0.330 & 14 & 15 & 1 & 1 & 1 & 1 & 1 & 2 \\
\hline Q9 & 0.965 & 14 & 16 & 2 & 2 & 1 & 1 & 2.25 & 2 \\
\hline \multicolumn{10}{|l|}{$T$ test } \\
\hline Q10 & 0.633 & 13 & & 90 & 90 & 72.5 & 85 & 94 & 93.75 \\
\hline
\end{tabular}


2013. Informed consent was obtained from all patients for being included in the study.

\section{RESULTS}

The results of the statistical analysis show no significant differences between the patient group that viewed the video (group A) and the usual verbal instruction group (group B). $P$ values for all of the results are greater than 0.05. Table 2 shows, for each question, the $p$ value, sample size for groups $A$ and $B$, the median value of response for groups A and B, and the 25th and 75th percentiles for groups $\mathrm{A}$ and $\mathrm{B}$.

\section{DISCUSSION}

Proper pre-treatment instruction is vital in alleviating anxiety and apprehension about the use of 5-FU cream for treatment of actinic keratoses. Furthermore, this may improve management of diffuse actinic damage and possibly decrease the incidence of squamous cell carcinomas developing from actinic keratoses. In the present study, viewing an educational video prior to treatment did not lead to statistically significant differences in patient satisfaction and treatment completion rates when compared to usual verbal instruction.

Our results show no significant differences between the video and usual verbal instruction group, with regards to the questionnaire responses (Table 1). For example, when asked: "How satisfied are you with the doctors who treated your pre-cancers (actinic keratoses)?" in Q6, 10 of $12(83 \%)$ selected "very satisfied" in the video group, while 18 of 18 (100\%) selected "very satisfied" in the usual verbal instruction group. This difference was not found to be statistically significant. Additionally, when asked: "Did you complete your treatment as instructed by your dermatologist?" in Q8, 12 of
12 (100\%) missed doses 3 days or less during the treatment period in the video group, whereas 17 of 18 (94\%) missed doses 3 days or less in the usual verbal instruction group. Again, this difference was also not found to be statistically significant.

Our findings present a few implications. First of all, the patient educational video may provide the same high level of satisfaction as the usual verbal instruction group, as evidenced by the median Q10 score of 90 out of 100 , where 100 represented the highest level of general satisfaction, in both the video and usual verbal instruction group. Given this, one can argue that showing patient education videos as a substitute to usual verbal instruction in the office will yield identical treatment outcomes in the context of a complicated medical regimen as in the case of 5-FU treatment for actinic keratosis. Not only may this yield identical treatment outcomes and patient satisfaction, but the option of employing patient educational media such as video yields increased clinic efficiency and opportunities for a multidisciplinary approach to pre-treatment education, as various members of the care team including nursing staff and medical assistants can play a key role in delivering the media to the patient in the office setting.

Potential limitations to the study are as follows: There is a small sample size and results do not reach statistical significance. Also, the method of MD instruction was not standardized. The video group received an introduction to the concept of 5-FU treatment by the treating MD, which was not standardized or scripted across providers, and multiple MDs were involved in the instruction process for both groups. This introduction was understood to be significantly abbreviated compared to the usual verbal instruction given to the control 
group. This introduction may be perceived as full MD counseling in members of the video group and leaves the meaning of an introduction to 5-FU up to interpretation by the provider, leading to variation regarding the depth and detail of this treatment introduction. Furthermore, given that the questionnaires were not anonymous as they were performed via telephone with an MD provider, the participants may have felt a desire to please the MD by responding in a generally affirmative manner regarding their level of satisfaction. A fully anonymous modality for feedback might yield more accurate results in a future study.

\section{CONCLUSION}

No significant differences in patient satisfaction were found between the patient group that watched the video and the patient group that did not watch the video. A larger sample size could have produced a more robust analysis. We speculate that this study may establish a foundation for subsequent studies that may affect the broader medical community and promote development of educational videos for additional arenas of patient care, including wound care and medications requiring self-injection.

\section{ACKNOWLEDGMENTS}

No funding or sponsorship was received for this study or publication of this article. All named authors meet the International Committee of Medical Journal Editors (ICMJE) criteria for authorship for this manuscript, take responsibility for the integrity of the work as a whole and have given final approval for the version to be published.
Disclosures. O. N. Agbai, P. Davari, J. Johnson, A. Armstrong and N. Fazel have nothing to disclose.

Compliance with Ethics Guidelines. All procedures followed were in accordance with the ethical standards of the responsible committee on human experimentation (institutional and national) and with the Helsinki Declaration of 1964, as revised in 2013. Informed consent was obtained from all patients for being included in the study.

Open Access. This article is distributed under the terms of the Creative Commons Attribution-NonCommercial 4.0 International License (http://creativecommons.org/licenses/ by-nc/4.0/), which permits any noncommercial use, distribution, and reproduction in any medium, provided you give appropriate credit to the original author(s) and the source, provide a link to the Creative Commons license, and indicate if changes were made.

\section{REFERENCES}

1. Makki D, Alameddine M, Al Khateeb H, Packer G. The efficacy of patient information sheets in wrist arthroscopy: a randomised controlled trial. J Orthop Surg (Hong Kong). 2011;19:85-8.

2. Idriss NZ, Alikhan A, Baba K, Armstrong AW. Online, video-based patient education improves melanoma awareness: a randomized controlled trial. Telemed J e-health Off J Am Telemed Assoc. 2009;15:992-7.

3. Armstrong AW, Idriss NZ, Kim RH. Effects of video-based, online education on behavioral and knowledge outcomes in sunscreen use: a randomized controlled trial. Patient Educ Couns. 2011;83:273-7.

4. Armstrong AW, Alikhan A, Cheng LS, Schupp C, Kurlinkus C, Eisen DB. Portable video media for presenting informed consent and wound care instructions for skin biopsies: a randomized controlled trial. Br J Dermatol. 2010;163:1014-9. 
5. Armstrong AW, Kim RH, Idriss NZ, Larsen LN, Lio PA. Online video improves clinical outcomes in adults with atopic dermatitis: a randomized controlled trial. J Am Acad Dermatol. 2011;64:502-7.

6. Migden M, Chavez-Frazier A, Nguyen T. The use of high definition video modules for delivery of informed consent and wound care education in the Mohs Surgery Unit. Semin Cutan Med Surg. 2008;27:89-93.

7. Redzuan NS, Engkasan JP, Mazlan M, Freddy Abdullah SJ. Effectiveness of a video-based therapy program at home after acute stroke: a randomized controlled trial. Arch Phys Med Rehabil. 2012;93:2177-83.

8. Askew DA, Mickan SM, Soyer HP, Wilkinson D. Effectiveness of 5-fluorouracil treatment for actinic keratosis-a systematic review of randomized controlled trials. Int J Dermatol. 2009;48:453-63.

9. Tierney EP, Eide MJ, Jacobsen G, Ozog D. Photodynamic therapy for actinic keratoses: survey of patient perceptions of treatment satisfaction and outcomes. J Cosmet Laser Ther Off Publ Eur Soc Laser Dermatol. 2008;10:81-6. 